\title{
Modeling of divertor particle and heat loads during application of resonant magnetic perturbation fields for ELM control in ITER*
}

\author{
O. Schmitz ${ }^{\mathrm{a}}$, M.Becoulet ${ }^{\mathrm{b}}$, P. Cahyna ${ }^{\mathrm{c}}$, T.E.Evans ${ }^{\mathrm{d}}$, Y. Feng $^{\mathrm{e}}$, \\ H. Frerichs ${ }^{\text {a }}$, A. Kischner ${ }^{\mathrm{a}}$, A.Kukushkin ${ }^{\mathrm{f}}$, R. Laengner ${ }^{\mathrm{a}}$, T.Lunt ${ }^{\mathrm{e}}$, \\ A.Loarte ${ }^{\text {f }}$, R.Pitts $^{\mathrm{f}}$, D. Reiser ${ }^{\mathrm{a}}$, D. Reiter ${ }^{\mathrm{a}}$, G. Saibene ${ }^{\mathrm{g}}, \mathrm{U}$ Samm $^{\mathrm{a}}$ \\ ${ }^{a}$ Forschungszentrum Jülich, IEK-4, Association EURATOM-FZJ, Jülich, Germany \\ $b_{C E A / I R F M}$, Cadarache, 13108 St. Paul-lez-Durance Cedex, France \\ cIPP AS CR, Za Slovankou 3, 18200 Prague 8, Czech Republik

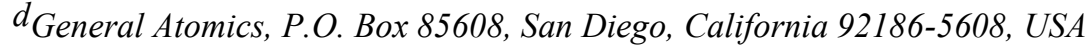

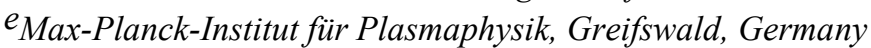 \\ $f$ ITER Organization, Route de Vinon sur Verdon, 13115 Saint Paul Lez Durance, France \\ $g_{\text {Fusion for Energy Joint Undertaking, Barcelona, Spain }}$
}

\begin{abstract}
First results from three-dimensional modeling of the divertor heat and particle flux pattern during application of resonant magnetic perturbation fields as ELM control scheme in ITER with the EMC3Eirene fluid plasma and kinetic neutral transport code are discussed. The formation of a helical magnetic footprint breaks the toroidal symmetry of the heat and particle fluxes. Expansion of the flux pattern as far as $60 \mathrm{~cm}$ away from the unperturbed strike line is seen with vacuum RMP fields, resulting in a preferable heat flux spreading. Inclusion of plasma response reduces the radial extension of the heat and particle fluxes and results in a heat flux peaking closer to the unperturbed level. A strong reduction of the particle confinement is found. 3D flow channels are identified as a consistent reason due to direct parallel outflow from inside of the separatrix. Their radial inward expansion and hence the level of particle pump out is shown to be dependent on the perturbation level.
\end{abstract}

JNM keywords: diffusion, physical properties, theory and modeling,

PSI-17 keywords: $\quad$ ELM control, stochastic boundary, edge modeling ITER

PACS: $\quad$ 52.25.Fi, 52.55.Fa, 51.60.+a

*Corresponding author address: Wilhelm-Johnen-Straße, D-52425 Jülich, Germany

*Corresponding author E-mail: o.schmitz@fz-juelich.de

Presenting author: Oliver Schmitz

Presenting author E-mail: o.schmitz@fz-juelich.de 


\section{Introduction}

A set of in-vessel magnetic control coils is being implemented into the ITER base line design. One main purpose of this coil set is the application of resonant magnetic perturbation (RMP) fields for control of type-I edge localized modes (ELMs)[1]. Such fields have been shown on various devices to suppress or mitigate the ELM induced, transient heat and particle loads to the divertor and first wall. During ELM suppression obtained so far in DIII-D [2] and strong mitigation observed at ASDEX-Upgrade [3], a striation of the divertor heat and particle fluxes is measured [4-6]. This has been seen also in ELM control experiments at NSTX [7], MAST and JET [8]. Recently direct imaging of the helical lobe structure of the perturbed separatrix causing these striated patterns have been obtained at MAST [9] and DIII-D [10]. These measurements provide strong evidence for the fact that formation of a three-dimensional (3D) plasma boundary is a quite generic feature of application 3D magnetic control fields. Therefore it has potentially significant consequences for the divertor erosion-deposition balance and material migration during ELM control by RMP fields in ITER. The plasma wall interaction in the divertor is likely being transformed from an axisymmetric assumption into a 3D situation [11]. Therefore it is important to assess the impact of $3 \mathrm{D}$ control fields in the divertor heat and particle fluxes at ITER and compare the results with the design criteria based on 2D transport analysis. However, this represents a significant challenge, as several basic assumptions for such 3D transport modeling are yet unclear even for present devices. This involves on the one hand response of the ITER plasma as highly conductive media and on the other hand the particular transport model used in the high temperature plasma at very low collisionality. We approach the problem by using existing tools as far as the transport model is concerned and study a case with plasma response from non-linear MHD modeling included. 
The paper is organized as follows: in section two the magnetic field input for the modeling, the resulting magnetic field topology and the assumptions for the edge transport and divertor flux modeling will be introduced. In section three, results from 3D modeling of divertor heat and particle fluxes are shown for an exemplary sequence of no-RMP, vacuum RMP and screened RMP fields. This provides a framework for the actual heat loads expected with RMP at various levels of plasma response (vacuum vs. strong screening). In section four a potential reason for the particle pump out observed within the modeling equations of the code is introduced and the impact on the divertor heat flux assessment with RMP fields is discussed. Section five gives a summary and conclusion.

\section{Modeling input and magnetic field topology}

In this contribution results from 3D plasma fluid transport and kinetic neutral transport modeling with the EMC3-Eirene code are presented. The EMC3 code [12] solves a set of Braginskii fluid equations (Particle, Energy and Momentum) using a Monte-Carlo method iteratively with a 3D kinetic neutral transport code EIRENE [13]. EIRENE supplies the particle, energy and momentum sources from neutrals released by the divertor elements. The code was originally developed for application to stellarators but recently adapted to 3D modeling of the plasma edge transport during RMP ELM suppression experiments in DIII-D [14]. A benchmark between EMC3-EIRENE and 2D modeling of the divertor conditions at ITER with B2-EIRENE [15] was performed. The physics captured in both codes is different, however, for this benchmark B2-Eirene was reduced to a comparable physics setup (see details in [15]). A satisfactory agreement was found on the level of a first time application of a code for a 3D situation in the ITER H-mode reference scenario. Agreement within 10\% in the upstream profiles, within $30 \%$ concerning downstream fluxes and a factor of two in the 
downstream density and temperature profiles was found [16]. This relates this modeling well to the 2D method used as standard divertor design guideline at ITER [15].

We consider for the plasma equilibrium of the standard $\mathrm{H}$-mode scenario at a plasma current $\mathrm{I}_{\mathrm{P}}=15 \mathrm{MA}$ with a toroidal field of $\mathrm{B}_{\mathrm{T}}=5.3 \mathrm{~T}$. The equilibrium used is constructed with a 4.5 keV pedestal electron temperature constraint yielding a moderate bootstrap current $\mathrm{I}_{\mathrm{BS}}$. Changing $\mathrm{I}_{\mathrm{BS}}$ yields a strong modification of the magnetic structure in the very edge and hence has a strong effect on the divertor heat and particle flux pattern [17]. The level of $I_{B S}$ chosen results in a kinetically stable edge plasma and represents a standard case for this ITER plasma equilibrium [18]. A 2D plot of this plasma equilibrium in terms of normalized pressure is shown in figure 1,a. The RMP field for ELM control is applied by a set of three rows of nine coils each as shown in figure 1,b. This is the actual design for the ITER ELM control set and allows a wide spectral variability. In figure 1,c the modeling domains at the inner (ISP) and outer (OSP) strike point are depicted as green lines in the divertor geometry used.

For this study, a toroidal mode number $n=3$ harmonic RMP field was applied. We study a case with a current distribution at highest coil current of 90kAt optimized for maximum extent of the vacuum magnetic island overlap [17]. This is compared with the solution obtained without RMP field and with a solution when the RMP field is screened by a plasma response. For this plasma response case a solution of the non-linear, cylindrical MHD code RMHD [19] was used for the same RMP setup. Here all resonant modes with $n=3,6$ and poloidal mode numbers $m<7$ are completely shielded while all modes with $m>=7$ have values close to the vacuum RMP level. This strong screening reduces the radial extension of the stochastic magnetic field line domain significantly as shown in figure 2 . Figure 2 , a shows the Poincare plots of the perturbed magnetic field line structure with vacuum field and figure $2, b$ with a screened RMP field. The red and green lines mark the position of the inner simulation 
boundary of the EMC3-EIRENE modeling domain. This direct comparison shows that the plasma screening reduces the stochastic layer extension from $30-35 \%$ of normalized poloidal flux in vacuum to a few percent only, taking plasma screening into account.

\section{Divertor fluxes with vacuum RMP and including plasma response}

The divertor regime addressed in this study is a fully attached, low recycling divertor. The input power is $\mathrm{P}_{\mathrm{H}}=100 \mathrm{MW}$ without impurity radiation power losses included. This represents an extreme case to test the upper bound for the heat fluxes into the $3 \mathrm{D}$ divertor footprint. Radial transport is described by radially constant perpendicular diffusion coefficients for particles $D_{\perp}=0.4 \mathrm{~m}^{2} / \mathrm{s}$ and heat $\chi_{\perp}=1.2 \mathrm{~m}^{2} / \mathrm{s}$ with a core particle source of $\mathrm{Q}_{\mathrm{C}}=2.0 \times 10^{21} \mathrm{~s}^{-1}$. This choice represents not an actual ITER operational point but intends to study a case of maximum heat fluxes at low density conditions. This setup is intended representing an upper bound of the 3D fluxes expected with RMP fields applied.

In figure 3 the resulting heat and particle flux patterns are shown for the three cases considered. In the upper row, heat fluxes are shown and particle fluxes in the lower row. The no-RMP case is depicted in the left column, the vacuum RMP solution in the middle and the screened RMP solution on the right. All flux patterns are analyzed at the outer strike point (OSP) with $\mathrm{y}$-axis as distance from the unperturbed strike point and the toroidal angle as $\mathrm{x}$ axis.

In general, formation of a $3 \mathrm{D}$ helical pattern is seen in the heat and particle fluxes when the RMP field is applied. This is a signature of formation of a 3D plasma boundary as measured reliably during ELM suppression for low collisionality plasmas in DIII-D [5,11]. However, in the experiment a weak heat inflow into the lobes reaching far away from the unperturbed SP is observed. This is not reproduced in the vacuum solution here, but an even filling of all lobes with a radial decay of the peak heat fluxes in the lobes similar to a scrape 
off layer (SOL) is seen. Including a plasma response in comparative modeling with EMC3EIRENE for ELM controlled discharges in DIII-D [20] have shown that this deviation between experiment and modeling can be reduced. The same trend is seen comparing the vacuum heat and particle fluxes (middle column in figure 3) with the ones obtained including a strong plasma screening (right column in figure 3). A clear reduction of the heat flux spreading is seen with a strongly reduced filling of the outer domains of each lobe. The peak heat fluxes are reduced from no-RMP to vacuum RMP by a factor of three and increase again to about $70 \%$ of the no-RMP value when plasma screening is included. This is shown in more detail in figure 4,a. Here, a cut along the outer strike line domain through the 2D heat flux pattern is shown. We clearly see a reduction of the peak heat flux from $31 \mathrm{MW} \mathrm{m}^{-2}$ for the noRMP solution (black dashed line) to $10 \mathrm{MW} \mathrm{m}^{-2}$ for the vacuum RMP case (green, solid line). Including plasma screening increases the heat flux peak value again to $16 \mathrm{MW} \mathrm{m}^{-2}$, with a strong reduction of the heat inflow into the outer lobe. This shows, that with application of vacuum RMP fields, the heat fluxes are preferably spread as far as $60 \mathrm{~cm}$ away from the outer strike line with an advantageous reduction of the peak heat flux. Including plasma screening reduces the heat flux spreading and yields a stronger peaking, which still is limited below the no-RMP solution.

A similarly strong striation is seen in the particle flux pattern as well. However, the divertor particle flux level increases by one order of magnitude going from the axisymmetric to the vacuum RMP solution. This is caused by the choice of the boundary condition. In DIIID, application of RMP fields for ELM control is frequently correlated with some level of particle pump out, i.e. a reduction of the plasma density [21]. For ITER, a reduction of the plasma density cannot be tolerated, as the fusion gain has to be achieved. Hence, in the simulations presented here the density at the inner simulation boundary (ISB) was maintained. Consequently any process yielding to enhanced outward transport and potentially as well a 
reduced neutral fueling efficiency will drive enhanced divertor recycling fluxes. With RMP fields applied, an increase in the radial particle transport leads to a flattening of the density profile and consequently - when the density at the ISB is fixed - an increase in the plasma pressure in the outer SOL region. Here, parallel transport becomes dominating and hence yielding a significant increase in the divertor particle flux due to the increased SOL pressure.

\section{Particle pump out and 3d flow channels}

This strong impact of the RMP field on the particle balance corresponds, in a simple single reservoir model as described in [22], to a reduction of the particle confinement time $\tau_{\mathrm{p}}$ by an order of magnitude for the vacuum RMP case relative to the no-RMP solution and by a factor of five for the screened solution relative to no-RMP. This remarkable reduction of the particle confinement is driven within the EMC3-EIRENE model by an inherent feature of the RMP induced magnetic field structure. The magnetic field lines in a chaotic magnetic topology, born from an external magnetic perturbation field which is aligned with the magnetic field line pitch angle, leave the plasma as correlated magnetic flow channels. This is discussed in comparison to experimental data in [23] for TEXTOR and from a generic vacuum field model in [14] for shaped plasmas in DIII-D. These correlated magnetic flux tubes connect into different toroidal directions towards the divertor targets and hence they establish counter streaming plasma flow channels. This is found in the ITER modeling result as shown in figure 5. Here the flow pattern is shown as Mach number since the flow is determined by the parallel pressure gradients and the Bohm criterion as boundary condition at the divertor surfaces. In consequence, a scrape off layer like parallel plasma flow along the magnetic flux tubes connecting within a few collisional mean free path lengths to the target is established. Figure 5, a shows the 2D cut of the plasma flow field for the vacuum RMP solution. A dense mesh of counter streaming flux tubes is seen extending inward over the 
entire perturbed domain for this high RMP amplitude level. Including a plasma screening reduces the magnetic field perturbation inside of 0.96 in normalized poloidal flux as shown in figure 2,b. This is reflected in cancelation of correlated flows inside of the separatrix for the flow field in the screened solution. This is shown in figure 5,b and results in a limitation of the 3D flow channels to the region close to the separatrix and outside. Therefore the particle pump out is reduced compared to the vacuum RMP solution.

However, as the screened case still allows field penetration up to four percent of the plasma radial domain, a strong effect on the neutral penetration fueling the plasma is expected. For the no-RMP solution, a large fraction of the ionization source is located in the domain of good flux surfaces. Since outward losses of ionized particles are determined by the perpendicular transport, a steep density gradient is expected at the separatrix. In case of RMP application - both vacuum case and screened case - open field lines exist within the ionization length scale and hence an additional, now parallel loss channel is established that flattens the density profile with increasing downstream density and decreasing downstream temperature. All these effects reduce the penetration probability of the recycling neutrals and hence reduce the fueling. Therefore, the significant increase in the divertor particle flux for both RMP cases is just a consequence of the higher "SOL" (perturbed open field line domain) plasma pressures resulting from the additional parallel particle channels and the density constraint at the ISB.

This increase in the divertor particle flux poses the question of how much of the heat flux reduction and preferable spreading discussed so far is a result of the modified recycling conditions with RMP applied. First, plasma-neutral interaction causes power losses $\Delta \mathrm{E}$, which are shown in table 4.1, last column. Obviously, this does not explain the flux spreading observed in the RMP cases where the neutral induced power losses even decrease in comparison to the no-RMP case in spite of an order of magnitude increase of the target 
particle flux. Second, the higher densities and lower temperatures in the RMP "SOLs" are indeed expected to spread heat fluxes because of the increased perpendicular-to-parallel transport ratio. This, together with an increase of the area wetted with SOL length field lines in the helical magnetic footprint pattern, results in the heat flux spreading observed.

\begin{tabular}{|l|l|l|l|}
\hline Scenario & $\int \boldsymbol{\Gamma}_{\text {target }}[\mathbf{k A}]$ & $\int \boldsymbol{q}_{\text {target }}[\mathbf{M W}]$ & $\Delta \boldsymbol{E}[\mathbf{M W}]$ \\
\hline No-RMP & 15.9 & 85.41 & 14.22 \\
\hline Screened RMP & 165.3 & 94.44 & 5.43 \\
\hline Vacuum RMP & 474.6 & 92.74 & 6.87 \\
\hline
\end{tabular}

Table 4.1: Overview of target heat and particle flux values for all three scenarios including the ionization losses to neutrals

\section{Conclusion and outlook}

The results from 3D fluid plasma and kinetic neutral transport modeling discussed in this paper shows that during application of RMP fields for ELM control at ITER a preferable spreading of the heat fluxes can be expected. A reduction of peak heat fluxes with a distribution over a large extend of the divertor surface was found for both RMP cases studied, i.e. a vacuum RMP solution and a solution with strong plasma screening for the RMP amplitude. The regime discussed was low density, linear recycling - i.e. low density divertor at $100 \mathrm{MW}$ of input power. This is not an actual operational point of ITER, but both magnetic topologies coupled with these boundary conditions represent a test case for the upper bound of the heat fluxes expected. The particle flux was found to increase with RMP field applied due to 3D flow channels streaming back refueling neutrals and opening a particle sink from inside of the unperturbed last closed flux surface.

These results are consistent within the fluid plasma transport and kinetic neutral transport package EMC3-EIRENE. However, the overall approach has specific limitations, which leave significant challenges to obtain a more reliable prediction for ITER for future 
work. First, the plasma response used was taken from a non-linear MHD model in cylindrical geometry. This requires several simplifications in the plasma equilibrium (q profile for instance), which have a strong impact on the magnetic input to the modeling. Second, the fluid approach used is known to overestimate parallel heat flux limits if treated in a classical way. Hence, the heat flux predictions should be taken with care as both major modeling constraints - magnetic topology, i.e. plasma response and transport model, i.e. fluid approach - determine the actual level of parallel heat transport. This parallel component is the quantity affected dominantly due to the open perturbed field lines generated by the RMP field applied. The level of field opening and the parallel heat conductivity are the primary ingredients for the heat fluxes discussed and are at the same time the most uncertain ones. However, if the parallel heat transport were reduced, a more local deposition of the heat flux would occur with still enhanced radial diffusion due to some level of stochastization at least in the very edge, e.g. for the screened case. So, the heat flux levels presented here represent at the moment the best upper bound estimate which can be obtained at present given the general challenge of 3D transport modeling.

\section{Acknowledgements}

*Work supported by F4E grant GRT-055(PMS-PE), by the U.S. Department of Energy under DE-FC02-04ER54698 and by the Grant Agency of the Czech Republic under grant P205/11/2341 


\section{References}

[1] A. Loarte et al., Proc. $23^{\text {nd }}$ IAEA FEC, Daegeon (Korea), ITR/1-4, 2010.

[2] Evans T.E., et al., Nucl. Fusion 48, 024002 (2008)

[3] Suttrop W., et al., Phys. Rev. Lett., 106, 225004 (2011)

[4] Evans T.E:, et al., J. Phys.: Conf. Series 7, 174 (2005)

[5] Schmitz O., et al., Plasma Phys. Control. Fusion., 50, 124029 (2008)

[6] Müller H.W., et al., Journ. Nucl. Mater., proceedings of PSI2012 (2012)

[7] Ahn J.-W., et al., Nucl. Fusion, 50, 045010 (2010)

[8] Nardon E., et al., Journ. Nucl. Mater., 415, 914-17 (2011)

[9] Kirk, A., et al., Phys. Rev. Letters, 108, 255003 (2012)

[10] Shafer, M.W., et al., Nucl. Fusion, 52, (2012)

[11] Schmitz O., et al., Journ. Nucl. Mater. 415, 886-893 (2011)

[12] Feng Y., et al., Nucl. Fusion., 45, (2005) 89-95

[13] Reiter D., et al., Fusion Science and Technology 47, (2005) 2, 172

[14] Frerichs H., et al., Nucl. Fusion 50, 034004 (2010)

[15] Kukushkin, A.S., et al., Fusion Eng. Design 86 (2011) 2865-2873

[16] Feng, Y., et al., 38 ${ }^{\text {th }}$ EPS Conference Strasbourg, June 27, 2011 ECA, Vol.35G, P1.071

[17] Evans, T.E., et al., "Modeling of vacuum magnetic field topology during RMP ELM control at ITER", ITER task agreement 2010, to be presented at IAEA 2012 conference, San Diego, CA, USA

[18] Casper T., et al., "Development of the ITER baseline inductive scenario", Nucl. Fusion 52, (2012), at press

[19] Becoulet M., et al., "Screening of resonant magnetic perturbations by flows in tokamaks", Nucl. Fusion 52, (2012), at press

[20] Frerichs, H., et al., "Impact of screening of resonant magnetic perturbations in 3D edge plasma transport simulations for DIII-D”, Phys. Plasmas, (2012), at press

[21] Unterberg E.A., et al., Nucl. Fusion 50, 034011 (2010)

[22] Schmitz O., et al., Journ. Nucl. Mater. 415, 886-893 (2009)

[23] Schmitz O., et al., Nucl. Fusion., 48, 024009 (2008) 


\section{Figure captions}

Figure 1: Normalized toroidal magnetic flux depicting the plasma equilibrium used for the modeling of the standard $\mathrm{I}_{\mathrm{P}}=15 \mathrm{MA}, \mathrm{B}_{\mathrm{T}}=5.3 \mathrm{~T}$ H-mode scenario for energy gain of $\mathrm{Q}=10$ (figure a). Figure b shows the actual design of the ELM control coils consisting out of three rows with nine coils each. Figure c shows a zoom into the ITER divertor depicting the extension of the modeling domains along the inner (ISP) and outer (OSP) strike line domains.

Figure 2: Comparison of magnetic field line structure for (a) a case with vacuum RMP field from 90kAt coil currents in a phase configuration optimized for maximum stochastic layer extension and (b) for a case with strong screening of the RMP modes. The stochastic layer width is reduced from more then $30 \%$ extend to a few percent only. This allows moving the inner simulation boundaries (green and red lines) outward reducing the radial extension of the modeling domain. Note the different radial ranges on both plots.

Figure 3: Divertor heat (upper row) and particle (lower row flux) densities for an axisymmetric solution (figures a and d), a solution with RMP field at maximum current amplitude of 90kAt (figure b and e, field line structure corresponding to figure 2,a) and a solution with strong plasma screening (figure $\mathrm{c}$ and $\mathrm{f}$, magnetic field topology corresponding to figure 2,b). Note the change of the color bar limits needed to highlight the order of magnitude changes in the particle and factor of three changes in the heat flux.

Figure 4: Profiles of heat (figure a) and particle (figure b) flux densities at toroidal angle of 20 degree (position of maximum radial extension of the helical stripes). The coordinate along the wall is measured as distance from the unperturbed strike line. Profile of an axisymmetric solution (dashed black line), one solution with vacuum RMP field at $90 \mathrm{kAt}$ (green profile, 
magnetic field line structure according to figure 2 ,a) and one solution with screened RMP field (red profile, field topology according to figure 2,b) is shown.

Figure 5: Poloidal cuts at 0 degree toroidally of the plasma flow field as Mach number. Figure a shows the flow field for a vacuum RMP field at 90kAt (magnetic field structure according to figure 2,a) and figure b as found for a screened RMP field (field structure according figure 2,b). 
Figures

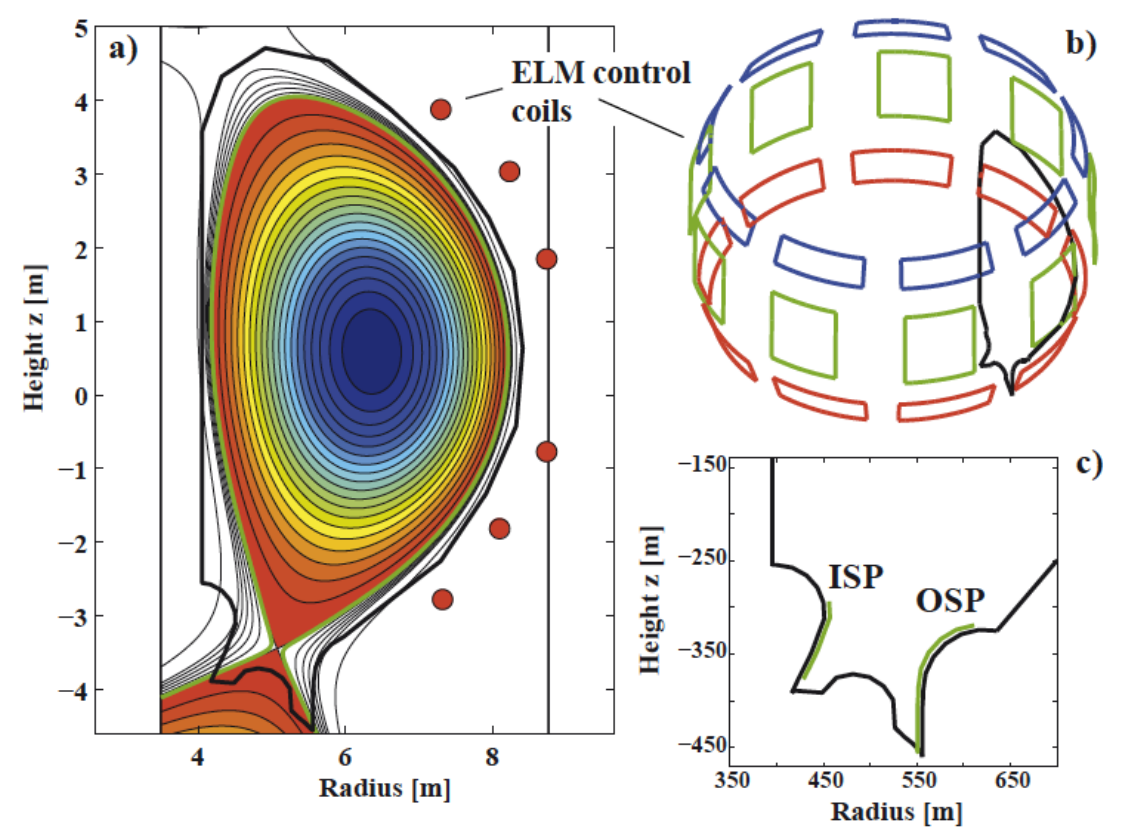

Figure 1 (figure labels increased) 


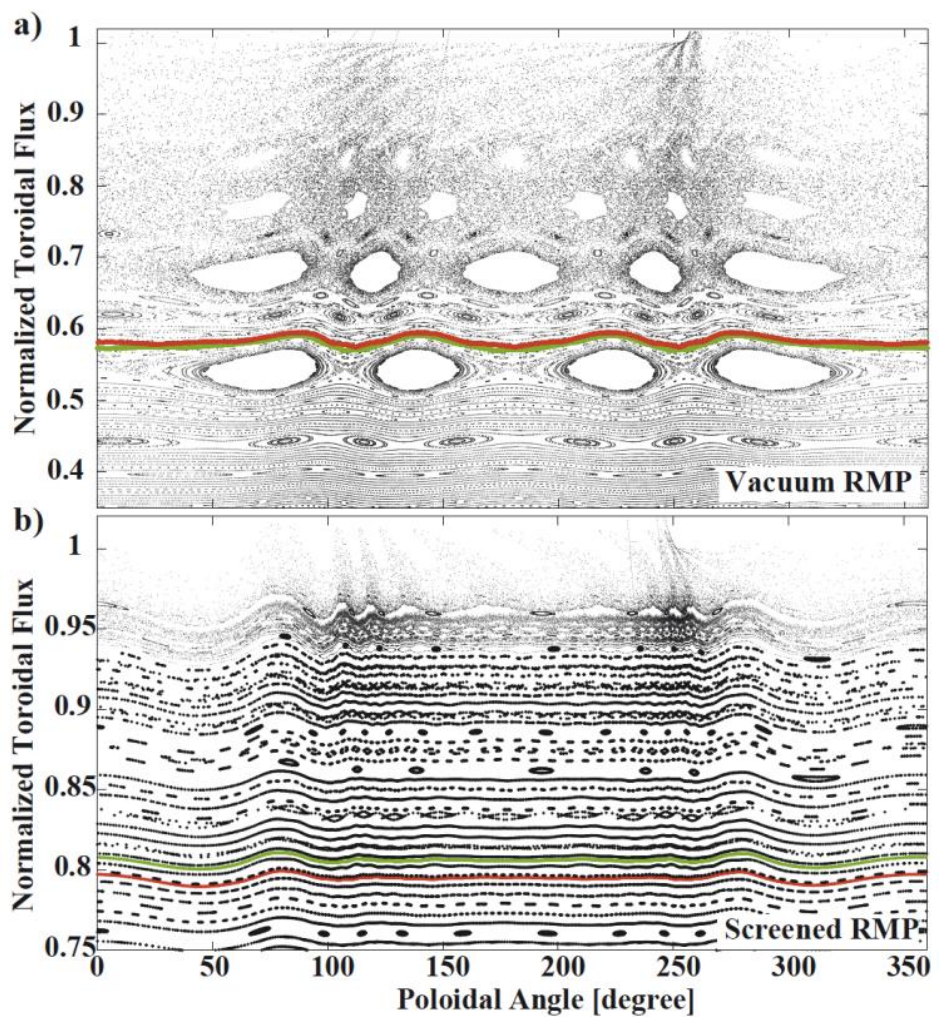

Figure 2 (figure labels increased) 


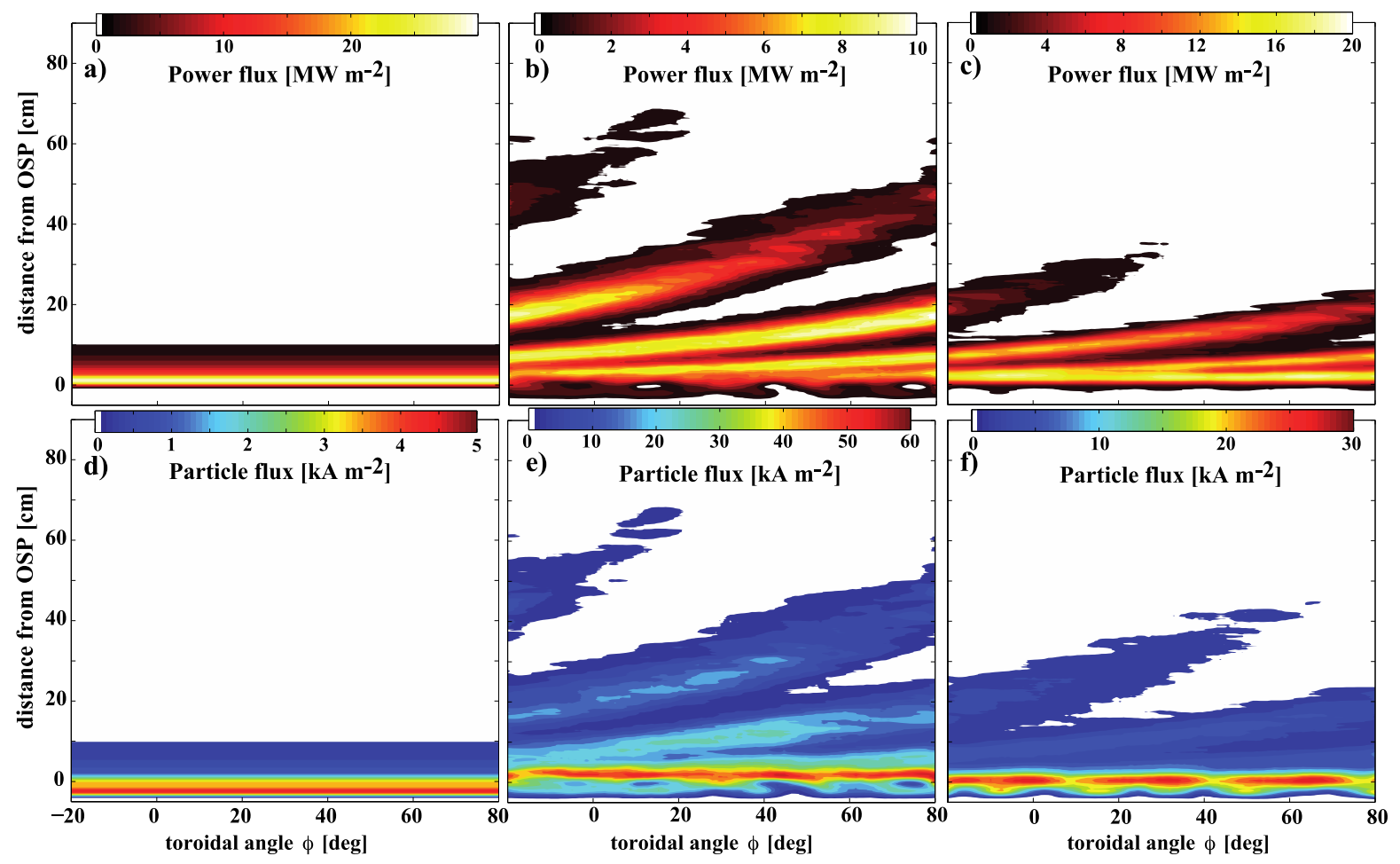

Figure 3 (figure labels increased)
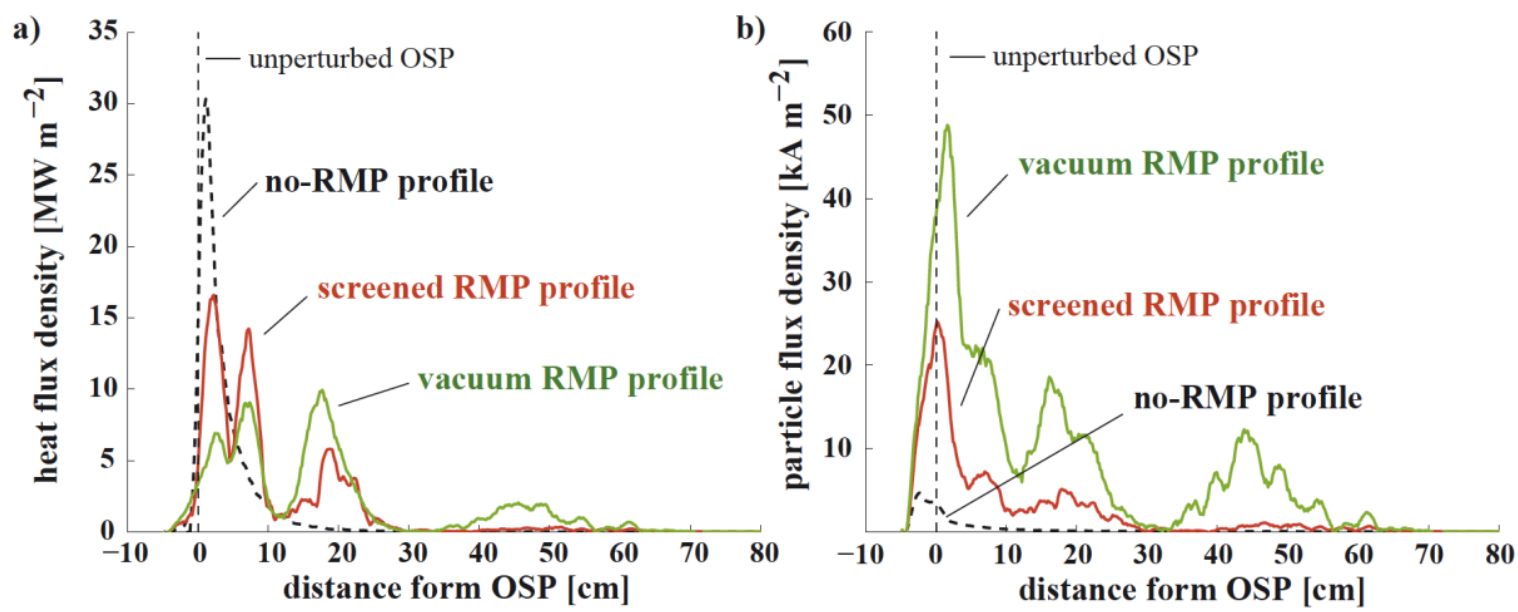

Figure 4 


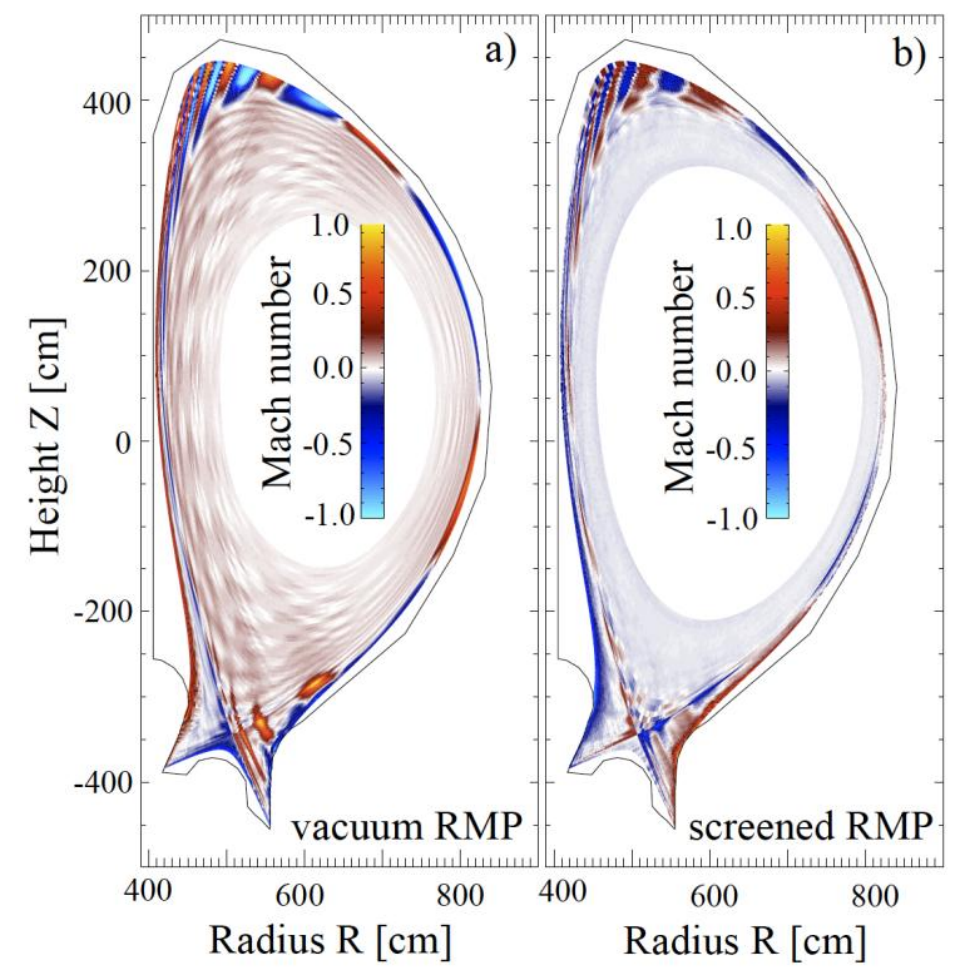

Figure 5 